\title{
A Bivariate Gaussian Model for Unexploded Ordnance Classification with EMI Data
}

\author{
David Williams, Member, IEEE, Yijun Yu, Levi Kennedy, Xianyang Zhu, and Lawrence Carin, Fellow, IEEE
}

\begin{abstract}
A bivariate Gaussian model is proposed for modeling spatially varying electromagnetic-induction (EMI) response of unexploded ordnance (UXO). This model is proposed for EMI sensors that do not exploit enough physics to warrant using the popular magnetic-dipole model currently commonly used. These two competing models are applied to measured EM61 sensor data at a real UXO site. UXO classification performance using the proposed bivariate Gaussian model is shown to be superior to an approach employing the magnetic-dipole model. Moreover, the bivariate Gaussian model requires no labeled training data, obviates classifier construction, and has fewer model parameters to learn.
\end{abstract}

Index Terms-Classification, dipole model, electromagneticinduction (EMI), Gaussian model, unexploded ordinance (UXO).

\section{INTRODUCTION}

$\mathbf{E}$ NVIRONMENTAL remediation of sites containing unexploded ordnance (UXO) is an expensive time-consuming endeavor. To avoid unnecessary excavation of non-UXO anomalies in this process, statistical classification techniques can be exploited. Specifically, a classifier can be constructed that is based on features extracted from raw measured sensor data. Toward this end, it has been shown that the spatially dependent magnetometer signal of UXO is well modeled by a simple magnetic dipole [1]. Moreover, a model for the electromagneticinduction (EMI) response of targets that generalizes this magnetometer model via a frequency-dependent magnetic dipole has also been developed [2]. The fit model parameters can then be used as features on which a classifier is constructed. Unfortunately, the quality of the EMI sensor that collects the data can limit the utility of the dipole model. If the collected sensor data do not exhibit a sufficient level of physics, the use of the sophisticated dipole model can be unwarranted.

The work in this letter-based on experience with real measured sensor data-proposes an elegantly simple alternative to the magnetic-dipole model for modeling EMI responses of anomalies. Specifically, a standard bivariate Gaussian model is employed to model the responses of interest. The use of this alternative model represents a fundamental difference in the data-modeling approach. Whereas the magnetic-dipole model seeks to exploit the underlying physics of EMI-based sensing, the bivariate Gaussian model instead focuses on the extraction of an EMI signature's shape and size information. Discrimina-

Manuscript received April 14, 2007; revised June 19, 2007.

The authors are with the Signal Innovations Group, Research Triangle Park, NC 27703 USA (e-mail: dpw @ siginnovations.com; yyu@ siginnovations. com; 1kennedy@siginnovations.com; xianyang@siginnovations.com; lcarin@ siginnovations.com).

Color versions of one or more of the figures in this paper are available online at http://ieeexplore.ieee.org.

Digital Object Identifier 10.1109/LGRS.2007.903972 tion between UXO and non-UXO is then based on the overall anomaly size. This letter will demonstrate that the proposed bivariate Gaussian model enjoys several advantages over the sophisticated magnetic-dipole model, which, in turn, make it an attractive alternative for UXO classification tasks.

The remainder of this letter is organized as follows. The magnetic-dipole model and the proposed bivariate Gaussian model for spatially varying EMI responses are presented in Sections II and III, respectively. Section IV describes the measured EMI sensor data used in this letter and then presents experimental results. This letter concludes in Section V with a discussion about the models and results.

\section{Magnetic-Dipole Model}

A model for the frequency- and time-dependent EMI response of UXO targets has been developed in [2]. Specifically, as a function of frequency, the magnetic-dipole moment $\boldsymbol{m}$ of a target is represented as

$$
\boldsymbol{m}=\mathbf{M H}^{\text {inc }}
$$

where $\mathbf{H}^{\text {inc }}$ denotes the incident (excitation) magnetic field, and $\mathbf{M}$ is the $3 \times 3$ dimensional magnetization tensor, relating the magnetic field to the magnetic-dipole moment. By taking the inverse Fourier transform of the frequency-domain EMI model, an analogous time-domain model can be obtained. This timedomain model is employed here because the data set used in this letter was collected by an EM61 sensor, which collects data in three time gates. For a UXO that is assumed to be rotationally symmetric with the axis of rotation along the $z$ direction (normal to the air-soil interface), the (time dependent) magnetization tensor can be expressed as a diagonal matrix

$$
\begin{aligned}
& \mathbf{M}=\operatorname{diag}\left[m_{p} \omega_{p} \exp \left\{-\omega_{p} t\right\}, \quad m_{p} \omega_{p} \exp \left\{-\omega_{p} t\right\},\right. \\
& \left.m_{z} \omega_{z} \exp \left\{-\omega_{z} t\right\}\right] \text {. }
\end{aligned}
$$

In (2), the terms $m_{z}$ and $m_{p}$ correspond to the magnetic-dipole moments of the target, which are directed perpendicular to and along the target's axis of rotation, respectively; the terms $\omega_{z}$ and $\omega_{p}$ correspond to EMI "resonant" frequencies (decay constants), while $t$ represents time.

If it is assumed that the EMI source that is responsible for the excitation magnetic field $\mathbf{H}^{\text {inc }}$ can be represented-as seen from the target - as a magnetic dipole with moment $\boldsymbol{m}_{s}$, then [2]

$$
\mathbf{H}^{\mathrm{inc}}=\boldsymbol{r} \frac{1}{2 \pi} \frac{\boldsymbol{m}_{s} \cdot \boldsymbol{r}}{\left|\boldsymbol{r}_{\mathrm{st}}\right|^{3}}
$$


where $\boldsymbol{r}_{\mathrm{st}}$ is the vector directed from the source to the target center, with $\boldsymbol{r}=\boldsymbol{r}_{\mathrm{st}} /\left|\boldsymbol{r}_{\mathrm{st}}\right|$ as the corresponding unit vector. Assuming sufficient proximity of the sensor's source and receiver coils, the total (time dependent) magnetic field observed at the sensor will be [2]

$$
\mathbf{H}^{\mathrm{rec}} \propto \frac{\boldsymbol{r}}{\left|\boldsymbol{r}_{\mathrm{st}}\right|^{6}} \boldsymbol{r}^{\mathrm{T}} \mathbf{U}^{\mathrm{T}} \mathbf{M U} \boldsymbol{r}
$$

where the proportionality constant depends on the strength of the dipole source $\boldsymbol{m}_{s}$ and the characteristics of the receiver.

The $3 \times 3$ unitary rotation matrix $\mathbf{U}$ rotates the fields from the coordinate system of the sensor to the coordinate system of the target, and $\mathbf{U}^{\mathrm{T}}$ transforms the dipole fields of the target (in the $\mathbf{M}$ coordinate system) back to the coordinate system of the sensor. Explicitly, the target orientation, in terms of the angles of the $\operatorname{target} \theta$ and $\phi$ with respect to the sensor coordinate system, is accounted for by

$$
\mathbf{U}=\left[\begin{array}{ccc}
\cos \phi & 0 & \sin \phi \\
0 & 1 & 0 \\
-\sin \phi & 0 & \cos \phi
\end{array}\right]\left[\begin{array}{ccc}
\cos \theta & \sin \theta & 0 \\
-\sin \theta & \cos \theta & 0 \\
0 & 0 & 1
\end{array}\right] .
$$

The EMI sensor employed in this letter measures the $z$-component of the magnetic field as a function of the position on the surface ( $z$ being normal to the air-soil interface). This measurement is subsequently fit to the model in (4) via a form of the Levenberg-Marquardt method [3]. Specifically, the parameters that the model inversion fits are the target position $(x, y$, and depth $z)$, the target orientation $(\theta$ and $\phi)$, the magnetic-dipole strengths $\left(m_{z}\right.$ and $\left.m_{p}\right)$, and the EMI resonant frequencies $\left(\omega_{z}\right.$ and $\left.\omega_{p}\right)$. Four parameters of the model $-m_{z}$, $m_{p}, \omega_{z}$, and $\omega_{p}$-are retained as features for the classification stage.

Because the model-inversion process is ill-posed, many solutions exist. To overcome the problem of local optima, several (model fitting) solutions are obtained, where each solution results from a random initialization of the model parameters. The final parameters of the model are taken to be those of the solution that minimizes the mean-square error between the measured and model-fit data.

\section{Bivariate GAussian Model}

The new modeling approach proposed in this letter does not attempt to model the complicated underlying physics of a UXO's response. The sensor data to be modeled and fit are instead viewed as a generic image. To determine the parametric form of the model to employ, the measured sensor data were examined visually. This data inspection led to the conclusion that a standard bivariate Gaussian model would accurately model the responses of interest.

The proposed bivariate Gaussian model is given by

$$
g(\boldsymbol{z})=(2 \pi)^{-1 / 2}|\Sigma|^{-1 / 2} \exp \left\{-\frac{1}{2}(\boldsymbol{z}-\boldsymbol{\mu})^{\mathrm{T}} \Sigma^{-1}(\boldsymbol{z}-\boldsymbol{\mu})\right\}
$$

where $z=\left[\begin{array}{ll}x & y\end{array}\right]^{\mathrm{T}}$ is the spatial position of the measured data, $\mu$ and

$$
\Sigma=\left[\begin{array}{ll}
\sigma_{11} & \sigma_{12} \\
\sigma_{12} & \sigma_{22}
\end{array}\right]
$$

are the free model parameters to be fit, and $g(\boldsymbol{z})$ is the spatially dependent (scalar) data measurement of an anomaly. In general, the response of an anomaly will be comprised of measured data at many different spatial positions. These data are jointly fit using the model in (6) (The sensor used in this letter collects data at each position in three different time gates. For bivariate Gaussian modeling purposes, we simply average these three sets of data.) Subsequently, from this model fit, a single scalar feature that represents the size of the anomaly is computed as

$$
\begin{aligned}
f & =\sigma_{11} \sigma_{22}|\Sigma|^{-1 / 2} \\
& =\sigma_{11} \sigma_{22}\left[\sigma_{11} \sigma_{22}-\sigma_{12}^{2}\right]^{1 / 2} .
\end{aligned}
$$

This size feature is used directly for classification purposes, in which large feature values are assumed to be associated with UXO.

\section{EXPERIMENTAL RESUlts}

\section{A. Sensor Data}

The utilities of the proposed bivariate Gaussian model and the competing magnetic-dipole model were evaluated using the measured EMI sensor data from the Fort Ord Seaside site in California. This site is divided into two distinct data sets: Seaside North and Seaside South. The spatially varying EMI data were collected using a Geonics EM61 MK2 sensor, using both the top and bottom coils. The EM61 MK2 was used in the wheel mode, and differential GPS was used for positioning. The line spacing was approximately $0.6 \mathrm{~m}$, and the point density along track was approximately 15 points $/ \mathrm{m}$.

A basic energy prescreener algorithm was applied to each site, which resulted in 1203 and 1072 discrete anomalies at the Seaside North and Seaside South sites, respectively. Of those anomalies, 82 were UXO at the Seaside North site, and 41 were UXO at the Seaside South site. The UXO types at the sites were 37-mm projectiles, hand grenades (M1A1, M69, and MKII), 40-mm M781, 75-mm projectiles, trench mortar, trench-mortar fuses, 3-in Stokes mortar, 3-in Stokes mortar fuses, 4-in Stokes mortar, and 4-in Stokes mortar fuses.

The sensor data associated with each anomaly were then used to perform inversion of the two models (bivariate Gaussian and magnetic dipole). The raw sensor data and the reconstructed signals using the fit model parameters are shown in Figs. 1-6 for six example anomalies. (For illustration purposes, the images corresponding to the raw sensor data and the reconstructed signals from the magnetic-dipole model were obtained by averaging across the data of the three time gates.)

\section{B. Experimental Setup}

The end result of the bivariate Gaussian model inversion process was the scalar size feature in (8). This feature was used directly for the final classification stage.

After performing the magnetic-dipole model inversion, four parameters $-m_{z}, m_{p}, \omega_{z}$, and $\omega_{p}$ from (2)—were retained for classification purposes. A kernel matching pursuit (KMP) [4] classifier was then constructed based on these four features. The popular KMP algorithm learns a linearly weighted kernel-based 


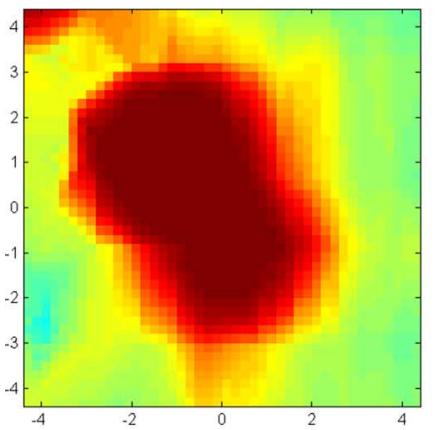

(a)

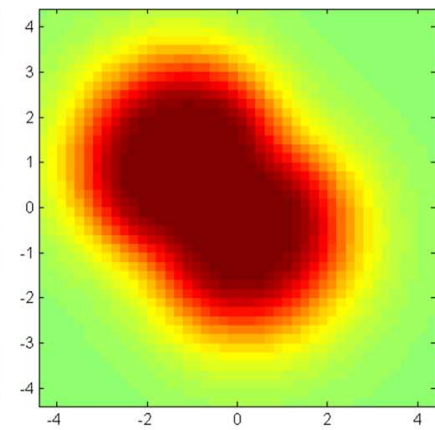

(b)

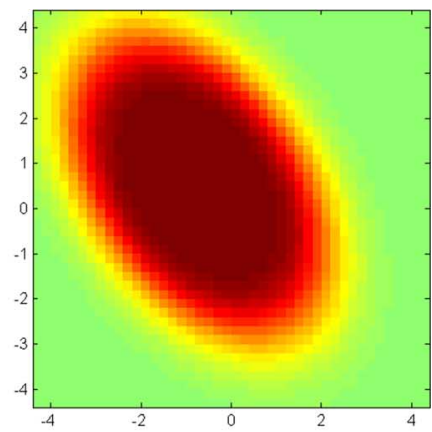

(c)

Fig. 1. Example (UXO) anomaly. (a) Raw EM61 sensor data. (b) Reconstructed signal using the fit dipole model parameters. (c) Reconstructed signal using the fit bivariate Gaussian model parameters.

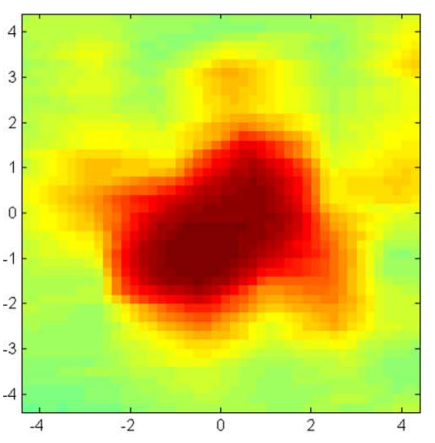

(a)

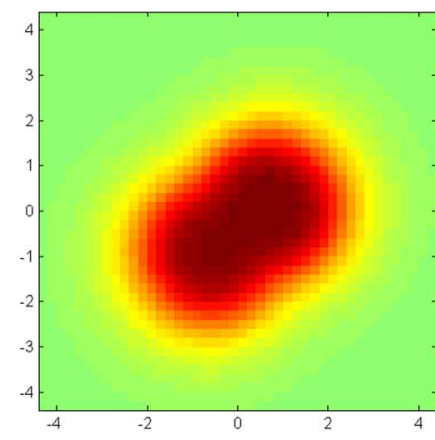

(b)

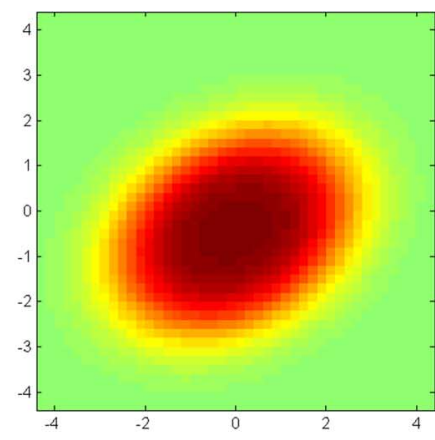

(c)

Fig. 2. Example (UXO) anomaly. (a) Raw EM61 sensor data. (b) Reconstructed signal using the fit dipole model parameters. (c) Reconstructed signal using the fit bivariate Gaussian model parameters.

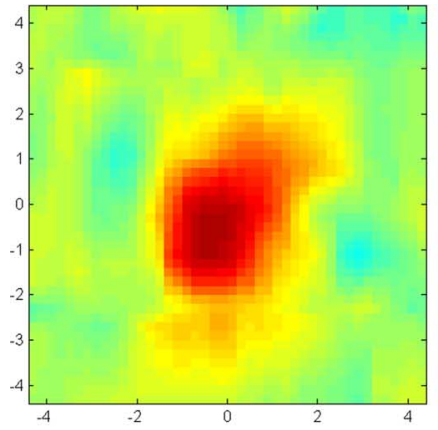

(a)

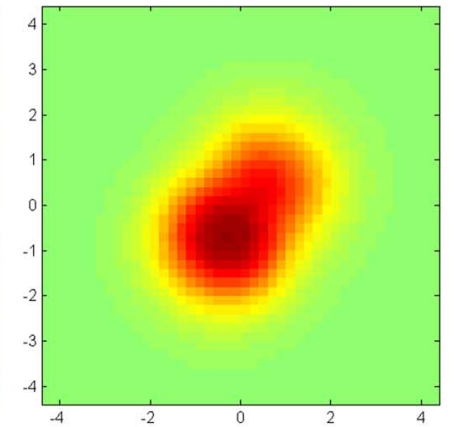

(b)

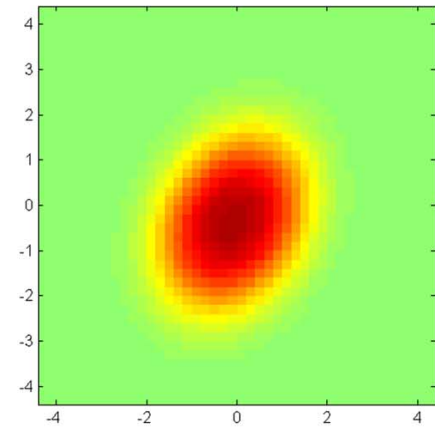

(c)

Fig. 3. Example (UXO) anomaly. (a) Raw EM61 sensor data. (b) Reconstructed signal using the fit dipole model parameters. (c) Reconstructed signal using the fit bivariate Gaussian model parameters.

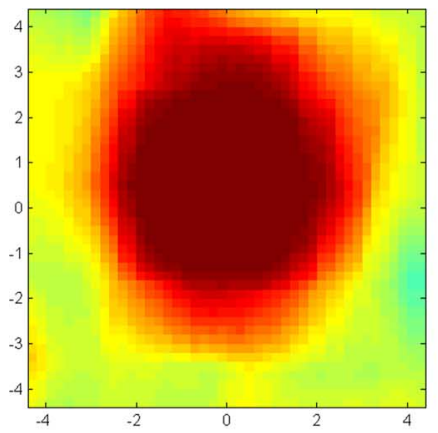

(a)

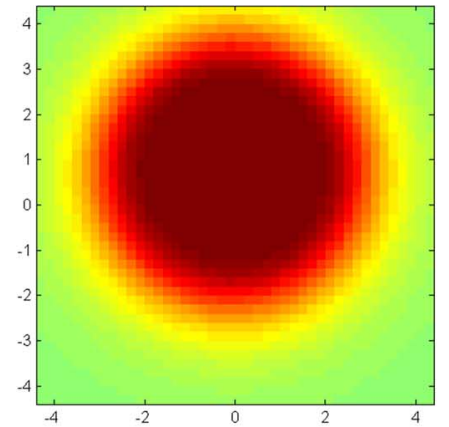

(b)

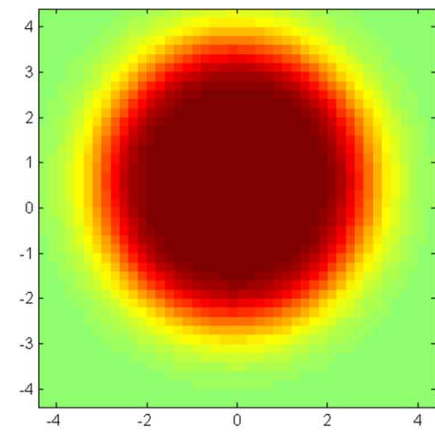

(c)

Fig. 4. Example (non-UXO) anomaly. (a) Raw EM61 sensor data. (b) Reconstructed signal using the fit dipole model parameters. (c) Reconstructed signal using the fit bivariate Gaussian model parameters. 


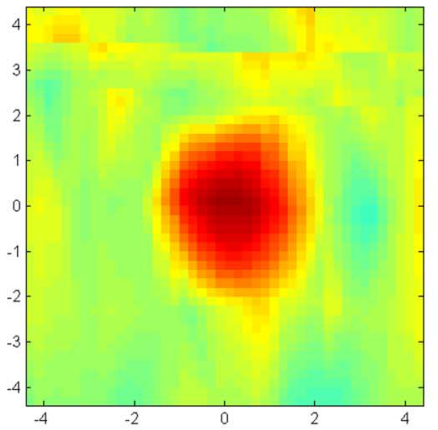

(a)

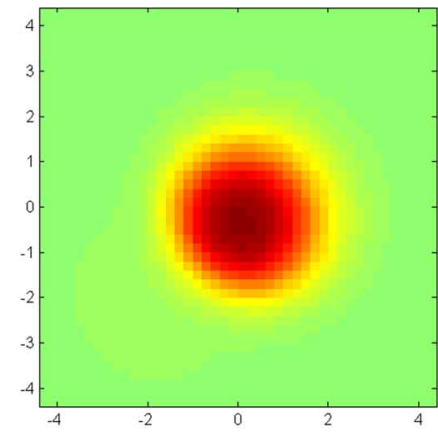

(b)

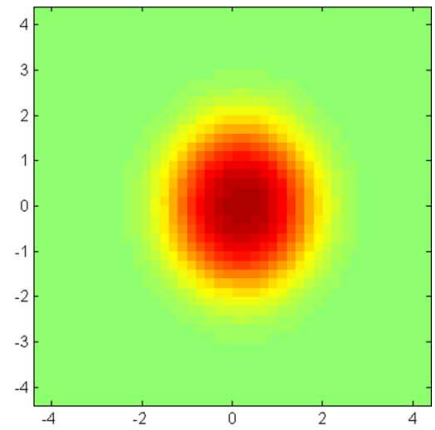

(c)

Fig. 5. Example (non-UXO) anomaly. (a) Raw EM61 sensor data. (b) Reconstructed signal using the fit dipole model parameters. (c) Reconstructed signal using the fit bivariate Gaussian model parameters.

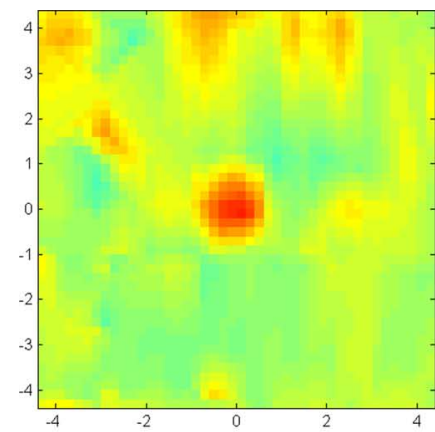

(a)

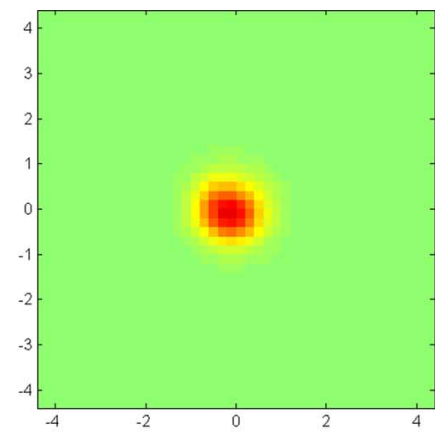

(b)

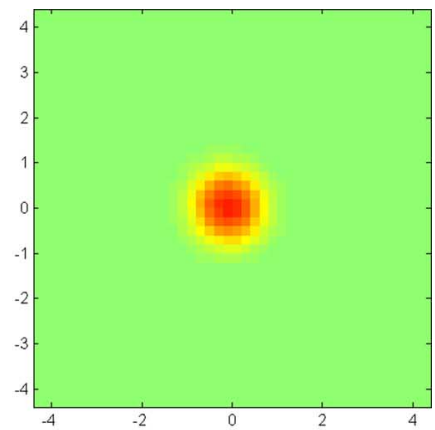

(c)

Fig. 6. Example (non-UXO) anomaly. (a) Raw EM61 sensor data. (b) Reconstructed signal using the fit dipole model parameters. (c) Reconstructed signal using the fit bivariate Gaussian model parameters.

regression function on which classification is based. Because KMP and the dipole model for which it is employed are not the main focus of this letter, we do not discuss the algorithm in detail here.

Leave-one-out training and testing was performed for this classification study employing KMP. (That is, one anomaly is withheld from the set used to train the KMP classifier; the heldout anomaly, treated as unlabeled testing data, is then evaluated by the constructed classifier to obtain its likelihood of being UXO. This process is then repeated until each anomaly is held out once in this manner.)

\section{Results}

Classification performance is expressed here in terms of receiver operating characteristic (ROC) curves. For the approach employing the bivariate Gaussian model, the ROC curves are generated by varying the threshold of the size feature (it is assumed that larger size-feature values are more indicative of UXO). A useful repercussion of performing classification that is based directly on the size feature is that any misclassified UXO will necessarily be small items, and any misclassified non-UXO will necessarily be large items. For the approach employing the magnetic-dipole model, the ROC curves are generated by varying the threshold of the output of the KMP classifier (the likelihood of being UXO). Fig. 7 shows the ROC curves for the two methods at the two sites. As can be seen from the figure, the approach employing the bivariate Gaussian model outperforms that of the magnetic-dipole model.
With regard to the ROC curves, it is important to remember that the two methods use their respective fit model parameters differently. Performing magnetic-dipole model inversion results in four parameters that are subsequently used for classification purposes. Specifically, a (KMP) classifier is constructed based on those four features. In contrast, the bivariate Gaussian model inversion results in a single (size) parameter, which is directly used for classification purposes (because the method results in only one parameter, a classifier need not be constructed). Therefore, even though the model-fit results using the magneticdipole model and the bivariate Gaussian model are very similar (as evidenced by Figs. 1-6), the resulting ROC curves associated with each method can exhibit differences. (The differences are not due to the two model fits being significantly different for certain anomalies; in fact, the anomalies shown in Figs. 1-6 are representative of how similar the two model fits typically are.)

We also present the classification results in terms of the area under an ROC curve (AUC). The AUC is given by the Wilcoxon statistic [5]

$$
\mathrm{AUC}=\frac{1}{M N} \sum_{m=1}^{M} \sum_{n=1}^{N} \mathbf{1}_{x_{m}>y_{n}}
$$

where $x_{1}, \ldots, x_{M}$ are the classifier outputs of data belonging to class 1 (UXO), $y_{1}, \ldots, y_{N}$ are the classifier outputs of data belonging to class 0 (non-UXO), and $\mathbf{1}$ is an indicator function. As a measure of classification performance, the AUC is a more 


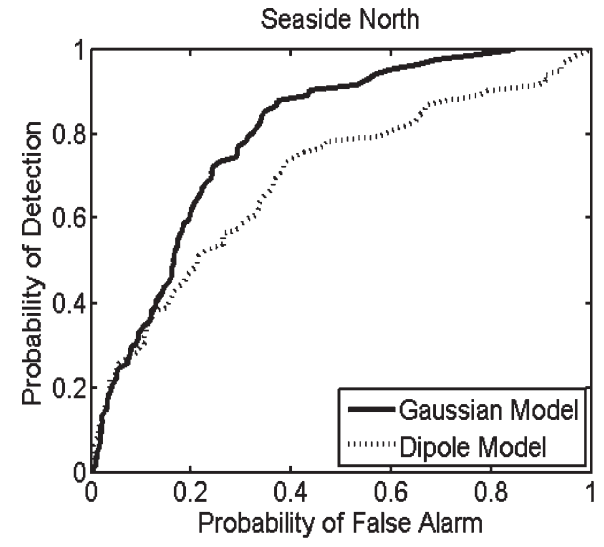

(a)

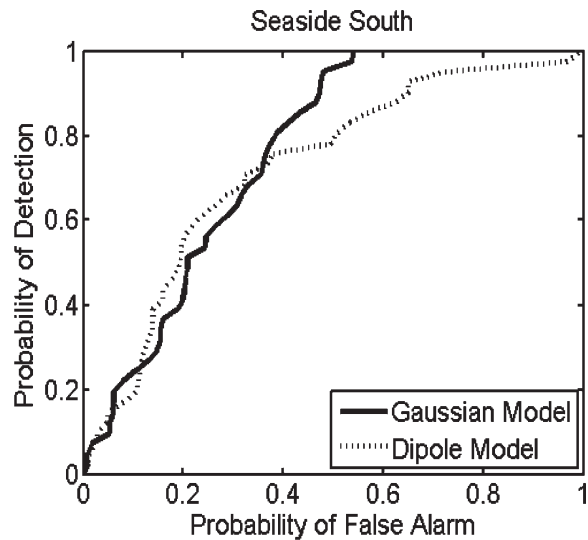

(b)

Fig. 7. Classification performance in terms of ROC curves for (a) Seaside North site and (b) Seaside South site.

TABLE I

AUC AsSOCIATED With THE ROC CURVES IN FIg. 7 AT THE Two SEASIDE Sites

\begin{tabular}{|c||c|c|}
\hline \multicolumn{1}{|c||}{ Site } & \multicolumn{2}{c|}{ Model } \\
Gaussian & Dipole \\
\hline \hline SEASIdE North & 0.7928 & 0.6894 \\
\hline SEASIde SOUth & 0.7533 & 0.7132 \\
\hline
\end{tabular}

useful quantity than accuracy (i.e., the fraction of classifications that are correct) when significant class imbalance exists, as it does in this data set. Moreover, the AUC can summarize the performance more compactly than an ROC curve. The AUC values for the two methods at the two sites are shown in Table I.

\section{CONClusion}

In this letter, we demonstrated that the classification performance on the measured EMI data using the simple bivariate Gaussian model exceeded that of the more sophisticated magnetic-dipole model. It should also be noted that these results were obtained when the magnetic-dipole model approach had over a thousand labeled anomalies on which to train a classifier, while the bivariate Gaussian model required no labeled data.

In addition to its superior classification performance, several additional reasons exist for adopting the proposed bivariate Gaussian model for UXO classification tasks. The magneticdipole model typically has many local optima because of the ill-posed nature of the model inversion. As a result, the fit model parameters may not be associated with the "correct" solution (that corresponds to the anomaly's true properties). However, even if the correct solution is selected, the timeconsuming model inversion process must still be conducted several times. When a UXO site has on the order of 1000 anomalies, these processing requirements are not negligible. In addition to the model inversion (feature extraction) process, a classifier must also be trained. This fact necessitates acquisition of labels (i.e., UXO or non-UXO) for some anomalies, a task that may be dangerous, costly, and time-consuming. In contrast, the proposed bivariate Gaussian model approach requires no labeled training data and obviates the construction of a classifier. Moreover, because the model has fewer parameters-five parameters compared to nine parameters in the dipole model-the inversion process is simpler and quicker (although having fewer model parameters allows less flexibility in the modeling process, it also likely reduces the number of (incorrect) local solutions).

In this letter, we compared the proposed bivariate Gaussian model to the magnetic-dipole model with a KMP classifier. It is important to note that the specific choice of (any reasonable) classifier would not significantly impact the classification performance on these data because the dipole-model features of the two classes (UXO and non-UXO) are heavily overlapping. This fact highlights another drawback of the magnetic-dipole model for the particular sensor employed: the lack of discriminability, based on dipole-model features, between UXO and non-UXO. The proposed bivariate Gaussian model avoids this problem by using anomaly size directly as the basis for discrimination between UXO and non-UXO.

If a more sophisticated EMI sensor that captured more of the physics of the problem was employed, the magnetic-dipole model may be valid. However, our experience and results suggest that the EM61 sensor does not exploit enough physics to warrant the use of the magnetic-dipole model. Instead, the proposed bivariate Gaussian model appears to be an appropriate alternative for UXO classification tasks with spatially varying EMI data.

\section{REFERENCES}

[1] C. Baum, Ed. Detection and Identification of Visually Obscured Targets, New York: Taylor \& Francis, 1998.

[2] Y. Zhang, L. Collins, H. Yu, C. Baum, and L. Carin, "Sensing of unexploded ordnance with magnetometer and induction data: Theory and signal processing," IEEE Trans. Geosci. Remote Sens., vol. 41, no. 5, pp. 1005-1015, May 2003.

[3] W. Press, B. F. S. Teukolsky, and W. Vetterling, Numerical Recipes in C: The Art of Scientific Computing, 2nd ed. Cambridge, U.K.: Cambridge Univ. Press, 1992.

[4] P. Vincent and Y. Bengio, "Kernel matching pursuit," Mach. Learn., vol. 48, no. $1-3$, pp. $165-187,2002$.

[5] J. Hanley and B. McNeil, "The meaning and use of the area under a receiver operating characteristic (ROC) curve," Radiology, vol. 143, no. 1, pp. 29-36, Apr. 1982. 